Philosophie ANTIQUE

\section{Philosophie antique}

Problèmes, Renaissances, Usages

$9 \mid 2009$

Néoplatonisme

\title{
Voula TSOUNA, The Ethics of Philodemus
}

New York, Oxford U. P., 2007, 350 p. ISBN : 978-0-19-929217-2.

Joëlle Delattre

\section{QpenEdition}

Journals

Édition électronique

URL : https://journals.openedition.org/philosant/2842

DOI : 10.4000 /philosant.2842

ISSN : 2648-2789

Éditeur

Éditions Vrin

\section{Édition imprimée}

Date de publication : 13 novembre 2009

Pagination : 224-228

ISBN : 978-2-7574-0124-8

ISSN : 1634-4561

\section{Référence électronique}

Joëlle Delattre, "Voula tsouna, The Ethics of Philodemus », Philosophie antique [En ligne], 9 | 2009, mis en ligne le 25 juillet 2019, consulté le 03 décembre 2022. URL : http://journals.openedition.org/ philosant/2842 ; DOI : https://doi.org/10.4000/philosant.2842

\section{(9) $\odot \Theta$}

Creative Commons - Attribution - Pas d'Utilisation Commerciale - Pas de Modification 4.0 International - CC BY-NC-ND 4.0

https://creativecommons.org/licenses/by-nc-nd/4.0/ 
blique ou encore en Ménon, 98a). De plus, Grabowski va peut-être un peu loin lorsqu'il prétend (p. 98) qu'il faut prendre le vocabulaire visuel de Platon au premier degré et non dans un sens métaphorique. Il semble clair que le lexique de la vue joue un rôle prépondérant tout au long de l'œuvre de Platon et il paraît difficile de lui nier toute valeur métaphorique, au moins dans la mesure où ledit lexique est amplement utilisé dans des passages ayant eux-mêmes un rôle illustratif comme, par exemple, les allégories ou les mythes. Finalement, même si Grabowski a sans doute raison de refuser aux Formes platoniciennes le statut d'universel, les représenter comme des particuliers n'est pas forcément la bonne solution. Si l'on veut critiquer la tendance à identifier les Formes de Platon à des universaux, il ne faut pas non plus les identifier à des particuliers, puisque ces deux termes sont liés au sein de la même articulation conceptuelle, laquelle n'est par ailleurs pas spécifiée explicitement dans la métaphysique de Platon. Plus encore, il faudrait demander à Grabowski quel genre de particuliers sont exactement les Formes du Bien et du Beau, Formes qu'il laisse de côté dans son étude au profit, par exemple, de celle du Lit. Or, il semble clair qu'il est plus aisé de se représenter comme un particulier la Forme du Lit que celle du Beau car la première se situe dans un rapport plus évident avec une imagerie visuelle que la seconde. Il faudrait donc trouver pour qualifier les Formes une autre voie qui dépasserait le clivage universel-particulier. Quand Grabowski affirme que le modèle doit absolument être un objet concret, cela n'est pas tout à fait exact. En effet, le modèle d'une maison peut très bien être le plan d'un architecte et non un prototype concret. Dans ce cas, il s'agit d'une entité abstraite qui peut jouer le rôle de paradigme dans la mesure où la maison réalisée ne sera qu'imparfaite par rapport à ce modèle. Dans l'alternative que proposait Grabowski concernant le statut du paradigme du mètre, Platon n'aurait sans doute pas tranché pour la solution du mètre étalon comme objet concret. À vrai dire, l'option d'une loi abstraite, scientifique ou autre, n'est en tout cas pas à éliminer a priori.

Luca PITTELOUd

Voula TsounA, The Ethics of Philodemus, New York, Oxford U. P., 2007, 350 p. ISBN : 978-0-19-929217-2.

L'éthique de Philodème de Gadara est aujourd'hui accessible, en langue anglaise, dans une présentation thématique qui met en perspective toutes les œuvres actuellement publiées de l'épicurien d'Herculanum, protégé du beaupère de César, Calpurnius Piso Caesoninus, et ami de Virgile et d'Horace. Les trois cent cinquante pages du livre de Voula Tsouna, The Ethics of Philodemus, offrent en effet enfin au public la réorganisation et le commentaire, en un seul volume, de larges et très nombreux extraits (pas moins de 150 références au Franc-parler de Philodème, par exemple) des grands textes éthiques de Philodème, qui ont été déchiffrés sur les papyrus carbonisés de la bibliothèque épicurienne d'Herculanum, découverte à la fin du XVIII ${ }^{\mathrm{e}}$ siècle. Ces textes ont pu être régulièrement réédités et traduits, depuis plus de trente ans, sous l'impulsion de Marcello Gigante et avec le soutien du Centro Internazionale per lo Studio dei Papiri Ercolanesi (CISPE), à Naples et dans plusieurs universités d'Europe et 
des USA ; mais il n'est pas facile d'en prendre une vue d'ensemble ni d'en saisir clairement les enjeux. Aussi faut-il saluer l'entreprise d'analyse et de compréhension, menée courageusement par notre collègue philosophe de l'Université de Californie à Santa Barbara.

Dans une brève introduction, l'auteur précise comment son «étude de l'ensemble des œuvres éthiques de Philodème» cherche à maintenir l'équilibre entre «le travail de détail sur les textes et l'attention donnée aux points philosophiquement intéressants ", de manière à s'adresser à la fois aux chercheurs en philologie classique et en philosophie antique. En effet, beaucoup des extraits cités ont été retraduits à nouveaux frais à partir du texte grec (parfois personnellement corrigé, entre crochets droits, avec le mot grec suppléé en note de bas de page, p. 35 par exemple) et amendés soit par rapport aux propres publications de l'A., antérieures ou en préparation, soit grâce à certains textes et traductions encore inédits, aimablement communiqués par leurs auteurs (par exemple D. Armstrong pour La Colère, et B. Henry pour La Mort).

Les quatre premiers chapitres du livre permettent de situer avec précision la pensée de l'épicurien Philodème par rapport aux grandes lignes de l'enseignement épicurien, et d'apprécier à la fois sa fidélité à Épicure (ce que d'aucuns appellent son orthodoxie) et la manière dont il sait, dans le sillage de Zénon de Sidon et de Démétrios Lacon, intégrer, voire « renverser» certains éléments de la controverse avec les académiciens et les stoïciens pour fortifier et enrichir la doctrine du Jardin.

Le chapitre préliminaire s'appuie sur deux textes principaux, [Choix et Rejets] (PHerc. 1251) et Épicure (PHerc. 1232 et 1289b), et sur le témoignage de Cicéron, à travers la démarche de Torquatus (Fin. I) soigneusement comparée avec celle de Philodème. L'A. cherche encore à améliorer la traduction de [Choix et rejets] de 1995 (p. 20) et habitue progressivement le lecteur à reconnaître les textes, qu'on les désigne avec l'abréviation de leur titre latin, en traduisant leur titre ou, plus techniquement, par un numéro de papyrus. Trois grands thèmes épicuriens classiques sont abordés : "plaisir et peine », qui impliquent une «tension entre le rôle de l'esprit et celui du corps dans la poursuite du plaisir», en lien avec le concept de limite ; «vices et vertus », puis « amitié ». Ils sont d'emblée présentés dans leur aspect controversé et problématique, pour amener le lecteur à se demander «dans quelle mesure l'interprétation d'Épicure par Philodème, sous l'effet conjugué des travaux de l'école et de l'impulsion zénonienne, cherchait à établir une orthodoxie épicurienne ».

Le deuxième chapitre traite des vices, des émotions et des «morsures » ou « pincements ». C'est l'occasion d'affirmer combien les analyses de Philodème, comparées à celles, postérieures, des écoles rivales (Sénèque, Plutarque, Lucien), sont manifestement «plus robustes du point de vue du fondement théorique, des principes méthodologiques et de la compréhension riche et sophistiquée du matériau » (p. 32). La notion de «disposition» (diathesis) et la distinction entre aspects internes et externes de la personne vicieuse sont présentes dans tous les cas, qu'il s'agisse de l'arrogance, de la flatterie, de l'envie et de la cupidité, de l'amour érotique ou de la peur de la mort. L'A., forte de sa connaissance très précise de traductrice de la plus grande part de ces textes, peut affirmer que «les 
vices se rencontrent souvent ensemble», ont la même "base cognitive » et surtout qu'ils correspondent plus à une expérience qu'à une connaissance. En effet, Philodème s'intéresse à ces comportements, certes pour les diagnostiquer et les traiter, mais surtout pour nous aider à nous en protéger (p. 37); d'où l'insistance sur «les importantes connexions» entre les dispositions morales de quelqu'un et sa manière d'interagir avec les autres; d'où aussi l'importance accordée aux images mentales, à la fois parce qu'elles accompagnent les passions violentes et «parce qu'elles sont utiles pour la thérapie» (p. 42). Concernant la catégorie particulière d'émotions appelée «morsure » (degmos) ou " pincement» (nugmos), l'A. montre comment Philodème se démarque des analyses stoïciennes (Chrysippe, Posidonios) en considérant ces accidents émotifs comme des émotions véritables, qui ne sont naturelles et involontaires que jusqu'à un certain point (p. 48), et restent influençables par la raison. Addition philodémienne importante à la psychologie morale et à l'éthique épicuriennes, ces notions seraient « un essai réussi pour rendre certains aspects de la doctrine épicurienne, en particulier le traitement de la colère et de la peur de la mort, acceptables pour un public plus large» (p. 51). Peut-être s'agit-il aussi de faire comprendre que, si le sage est susceptible, selon Épicure, d'éprouver plus d'émotions que la plupart d'entre nous (Diogène Laërce, X, 117), sa sagesse n'est nullement entravée par ce fait, au contraire (note 57 ).

Le troisième chapitre aborde davantage des problèmes de méthode. Philodème est, selon l'A., " éclectique » dans l'utilisation de ses sources (p. 52). Il compose ses œuvres éthiques de façon systématique et achevée, selon un même modèle, semble-t-il, mais qu'il sait adapter aux opposants critiqués et aux sujets traités. Par ailleurs, les clés de son approche empirique de l'éthique sont développées dans son autre livre Les Inférences. Et l'A. d'insister en particulier sur l'importance de l'epilogismos, procédure de type inductif consistant à partir d'une observation ordonnée des faits évidents pour en tirer «par similitude » des conséquences concernant des choses dont nous n'avons pas l'expérience directement (p. 55-56) ; le principe capital « pour inférer correctement» consiste précisément à observer avec prudence qu'aucune évidence n'entre en conflit avec le résultat du raisonnement (p. 58), lequel peut aussi s'appuyer sur de l'information de seconde main et sur l'enquête historique et géographique. Quant à la métaphore du philosophe médecin de l'âme, elle est classique dans l'école épicurienne ; elle est longuement discutée (p. 60-63) par rapport à l'interprétation qu'en donne M. Nussbaum, et en référence aussi à quelques passages précis du Franc-parler (p. 65). Le recours aux "premiers concepts» ou aux "préconceptions » s'avère lui aussi plus conforme aux exigences canoniques de l'épicurisme ancien que spécifiquement philodémien.

Dans le quatrième chapitre, la vue d'ensemble des types d'exercices et méthodes de traitement de l'âme malade, trouvés chez Philodème, est mise en perspective dans le cadre plus large des listes antiques comme celle du Compendium de Musonius Rufus ou celles qu'a transmises Philon d'Alexandrie et dont P. Hadot et R. Sorabji ont approfondi l'analyse. L'A. en propose un regroupement en quatre catégories: "Comment lire, comment discuter, comment vivre et comment mourir », qui permettent de se rendre compte de la grande parenté des 
pratiques épicuriennes avec celles des autres écoles philosophiques, y compris concernant le franc-parler (parrhesia, p. 78). Sous un autre angle, en effectuant un certain nombre de rapprochements avec Lucrèce, l'A. pointe les stratégies de détachement, de renforcement (par mémorisation et réécoute, p. 81), de révision de «ce qu'on met sous les mots » (relabelling) et de «mise sous les yeux » (p. 82). Si l'attention au présent et la considération correcte du passé et du futur sont bien constitutives d'une dimension «intemporelle» de la sagesse épicurienne, et au cœur des célèbres arguments de la symétrie et de l'identité, c'est le recours au «portrait moral» qui apparaît finalement comme plus spécifiquement philodémien, en particulier avec le souci de mettre en relief les traits et les manières du sage, par contraste (p. 87) avec celles et ceux des non-sages (même instruits) et des fous.

La deuxième partie du livre offre au lecteur une brillante mise en pratique des stratégies «comment lire et comment discuter» les principaux livres éthiques de Philodème, afin d'en tirer le maximum de matière et de sens pour faire comprendre clairement « comment vivre et comment mourir », très humainement, en authentique sage épicurien. Sans doute est-ce la raison pour laquelle la première place (ch. 5) est réservée au long ouvrage sur Le Franc-parler (avec en appendice quelques considérations sur chacun des trois petits livres La Gratitude, La Flatterie et L'Envie), tandis que les deux dernières places (ch. 9 et 10) sont occupées par La Colère (et le terrible désir de se venger qu'elle engendre) et $L a$ Mort (et les angoisses et frayeurs qu'elle génère). Entre ces extrêmes, prennent place naturellement trois chapitres intermédiaires, plus précisément consacrés au «comment vivre »: La Flatterie (et le désir de plaire), L'Arrogance (et les vices apparentés), L'Économie (la richesse et la gestion du patrimoine).

Chacun des livres de Philodème est présenté avec beaucoup de précisions techniques, comme nous l'avons déjà dit, mais surtout l'A. s'efforce d'en proposer une authentique réécriture ordonnée, structurée et exigeante, qui offre en même temps, au fil d'un commentaire suivi, de larges extraits soigneusement retraduits du contenu si lacunaire de ces exceptionnels volumina épicuriens carbonisés. Contentons-nous ici de deux remarques.

Pour ce qui est du Franc-parler, seul livre dont le titre ait été connu dans l'Antiquité, le chapitre est divisé en quatre sections qui permettent d'aborder la nature, le but et les sortes de parrhesia, ainsi que les circonstances dans lesquelles elle s'applique, puis les caractères des étudiants, et leurs réactions positives ou négatives aux critiques parrbésiastiques (flatterie, pouvoir, mais aussi manque de confiance et d'estime de soi sont des obstacles évidents à l'efficacité de la parrhesia, à laquelle souverains, femmes et vieillards [p. 119] opposeraient, selon Philodème, une résistance particulière), ensuite les dispositions des professeurs et les manières dont elles affectent l'usage qu'ils font de la parrhesia, enfin «les pratiques de confession et de correction », envisagées du point de vue du sage. La mise en ordre ainsi proposée par V. Tsouna, fort attrayante pour le lecteur d'aujourd'hui, ne correspond toutefois pas à l'ordre véritable des très nombreux fragments de ce papyrus particulièrement délicat à reconstruire; car l'authentique organisation du développement philodémien reste encore, à ce jour, à mettre en évidence. 
En revanche, les six subdivisions du chapitre consacré à La Colère rendent beaucoup plus fidèlement compte de l'ordre du texte philodémien, comme en témoignent d'ailleurs les références scrupuleusement notées à la fin de chacune des citations; elles proposent, de ce fait, une interprétation plus plausible et fiable, étant bien entendu, néanmoins, que le texte conservé ne représente que le dernier tiers environ du volumen complet, et qu'il est difficile d'imaginer le contenu perdu des deux tiers qui précédaient. Ces réserves sont simplement destinées à prévenir les risques de confusion entre l'effort louable de mise en ordre, exigible d'un exposé d'ensemble sur chacun des livres de Philodème, et l'intention véritable du maître épicurien de Campanie, dont l'état lacunaire ou trop mutilé des papyrus ne permet pas de percer facilement le secret.

Joëlle DELATTRE 